\title{
Custos de confinamento de bovinos de corte no município de Colômbia, SP
}

Feedlot costs of beef cattle in the city of Colômbia, SP

\author{
Guilherme Portes Silva ${ }^{1 *}$; Teodoro Leonardo Michelucci Contin²; Antonio Carlos Ramos dos Santos ${ }^{3}$
}

\begin{abstract}
1*ESALQ/USP - Dr. Ciência Animal e Pastagens - Av. Pádua Dias, 11 - 13.419-80 - Piracicaba, SP - Brasil < guilhermeps@yahoo.com.br>
${ }^{2}$ Banco do Brasil - Assessor de Agronegócios - Rua Vinte, 554 - Centro - 14.780-070 - Barretos, SP - Brasil

${ }^{3}$ ESALQ/USP - Departamento de Ciência Animal e Pastagens - Av. Pádua Dias, 11 - 13.419-80 - Piracicaba, SP - Brasil
\end{abstract}

\section{Resumo}

O sistema de terminação de bovinos em confinamento possibilita intensificar a produção animal, pois se aglomera um elevado número de animais em pequenas áreas. Os principais problemas enfrentados nesse sistema de produção são o elevado custo da atividade, a remuneração do peso e as incertezas do mercado. Nesse sentido, esse estudo teve como objetivos analisar os índices econômicos de um sistema de terminação de bovinos em confinamento e identificar os principais itens que oneram a atividade. Foram confinados 2.579 animais da raça Nelore e anelorados em uma fazenda localizada no município de Colômbia, estado de SP, por um período de 110 dias. Forneceram-se três tipos de alimentação, classificadas em dieta de adaptação, crescimento e terminação, com duração de 14, 40 e 56 dias, respectivamente. Foram determinados indicadores econômicos, tais como margem bruta, índice de margem de contribuição e ponto de equilíbrio. A atividade resultou numa receita total de R \$ 7,9 milhões. Os componentes do custo operacional efetivo que exerceram maior influência sobre os custos da atividade do confinamento foram a aquisição dos animais (72,08\%), alimentação (24,08\%), despesas diversas (1,45\%), mão-de-obra (2,57\%), frete (1,80\%), sanidade $(0,10 \%)$ e impostos $(0,04 \%)$. A análise resultou em lucro de $\mathrm{R} \$ 450.000$ e margem de lucro de $5,7 \%$. Os componentes do custo operacional efetivo que exercem maior influência sobre os custos da atividade de confinamento é a aquisição dos animais e a alimentação. Os índices econômicos apontaram que a atividade foi lucrativa e está se remunerando em curto prazo.

Palavras-chave: índices econômicos, pecuária de corte, terminação de bovinos

\begin{abstract}
Beef cattle feedlots make it possible to intensify meat production, since a large number of animals are concentrated in small areas. Presently, the mains problems faced by livestock farmer are the high cost of the activity, a return of the animal weight and uncertainties of the market. In this sense, the objective of this study was to analyze the economic indexes of a typical feedlot in Brazil and to identify the main items that affected the activity. A total of 2,579 Nellore bulls were confined in a farm located in the municipality of Colombia, state of Sao Paulo for a period of 110 days. Adaptation, growth and finishing diets were offered with a duration of 14, 40 and 56 days, respectively. Economic indicators, such as gross margin, contribution margin index and break-even point, were analyzed in a descriptive way and grouped into tables. The activity resulted in a total revenue of $\mathrm{R} \$ 7.9$ million. The components of the operational cost that have the greatest influence on the costs of the feedlot activity are acquisition of the animals $(67.36 \%)$, food $(22.46 \%)$, miscellaneous expenses $(5.07 \%)$, labor $(3.4 \%)$, freight $(1.49 \%)$, health $(0.09 \%)$ and taxes $(0.04 \%)$. The analysis resulted in a net profit of $\mathrm{R} \$ 450,000$ and a profit margin of $5.7 \%$. The components of the effective operational cost that exert the greatest influence on the costs of the feedlot activity are the acquisition of the animals and the feeding. The economic indexes indicated that the activity was profitable and is paying in the short term.
\end{abstract}

Keywords: economic indicators, beef cattle, finishing beef cattle

\section{Introdução}

O sistema de terminação de bovinos em confinamento consiste em instalar lotes de animais em piquetes ou currais com área restrita, onde os alimentos são fornecidos em cocho. O principal objetivo desse sistema de criação é intensificar o sistema de produção, favorecendo menor risco da atividade e maior lucratividade (Santos et al., 2017). No Brasil, a atividade de confinamento ganhou maior notoriedade no início da década de 80 , quando se passou a fornecer alimentos suplementares no período da seca (Wedekin et al., 1994), época de menor produção de forragem e de pior valor nutritivo, devido à limitação pelos fatores climáticos. No entanto, o uso de confinamentos se tornou cada 
vez mais expressivo, e atualmente os maiores Estados que realizam confinamento são Goiás $(26,43 \%)$ e Mato Grosso (25,42\%), seguidos por São Paulo (15,14\%), Mato Grosso do Sul (12,06\%) e Minas Gerais (10,60\%) (Assocon, 2012).

Dentre as vantagens de se confinar, destaca-se a programação da época de abate, animais abatidos com menor idade, possibilidade de se produzir carne de melhor qualidade, melhor rendimento de carcaça no abate e venda dos animais aos frigoríficos na entressafra, quando o preço da arroba é maior (Peixoto et al., 1989). Embora tenha ocorrido, nos últimos anos, um aumento considerável na produção brasileira de gado de corte, os custos de produção pressionaram as margens de lucro, especialmente em sistemas de confinamento (Lobato et al., 2014; Kamali et al., 2016), de forma que são de fundamental importância o levantamento dos custos e o planejamento financeiro da atividade. A compra de animais para reposição é o item que exerce maior influência sobre os custos do confinamento, seguido pelos demais componentes do custo operacional efetivo: alimentação, despesas diversas, mão-de-obra, sanidade e impostos fixos (Lopes e Magalhães, 2005). Outros autores reportaram que o custo de alimentação, em alguns casos, pode representar o maior custo de produção em sistemas de produção de carne bovina em confinamento, explicando 50\% (Koknaroglu et al., 2005) a 90\% (Albright et al., 1993) da variabilidade de lucro.

No entanto, Lopes e Magalhães (2005) destacam que para o êxito da atividade exigem-se conhecimentos em administração financeira, nutrição animal, manejo nutricional, produção de volumosos, aquisição de insumos e de animais de reposição. Adicionalmente, a pressão cada vez maior para intensificação dos sistemas de produção de alimentos, aliado aos custos de insumos cada vez maiores, torna-se essencial fazer análises de índices econômicos para monitoramentos dos sistemas de produção e tomada de decisões que garantem o sucesso da atividade (Sartorello et al., 2018; Tang et al., 2017).

Dessa forma, objetivou-se com este estudo analisar os índices econômicos de um sistema de terminação de bovinos de corte em confinamento e identificar os principais itens que oneram a atividade, no município de Colômbias, SP.

\section{Material e Métodos}

Os dados analisados foram provenientes de um sistema de terminação de bovinos em confinamento, localizado no município de Colômbia, noroeste do estado de São Paulo, Brasil. A propriedade possuía cerca de 800 hectares, sendo que cinco hectares eram destinados às instalações de confinamento, como currais, piquetes e bebedouros. Foram utilizados 2.579 animais, com duração de 110 dias, correspondentes aos meses de junho a outubro de 2015. A região era de fácil acesso à aquisição de alimento, como o bagaço de cana-de-açúcar, a polpa cítrica e os subprodutos de grãos, os quais foram utilizados na formulação de dieta para os animais.

Considerou-se, para a análise dos índices econômicos, a área de cinco hectares. Os piquetes de terminação não possuíam cobertura e o piso era de terra batida compactada. Eram 20 divisões com aproximadamente $2.500 \mathrm{~m}^{2}$. Trabalhou-se com área mínima por animal de $20 \mathrm{~m}^{2}$. A área do curral de manejo era de 425 $\mathrm{m}^{2}$. O Barracão (galpão e escritório anexados) de armazenamento de máquinas, implementos e produtos possuía cerca de $500 \mathrm{~m}^{2}$ e as instalações eram suficientes para a condução da atividade.

Os animais confinados, constituídos de bois inteiros (não castrados) da raça Nelore e anelorados (mestiços), eram provenientes dos estados de Goiás, Minas Gerais e São Paulo e o transporte foi feito em caminhões terceirizados. A idade média dos bovinos era de 25 meses. O peso médio dos animais, na entrada do confinamento, era de $382 \mathrm{~kg}$. Estes foram submetidos ao manejo sanitário, sendo devidamente vacinados e vermifugados.

Os insumos foram adquiridos na região e na época de maior oferta de cada alimento, e os produtos ensacados foram armazenados em galpão fechado. Os produtos de origem agrícola, tais como farelo de amendoim, polpa cítrica e bagaço de cana, eram armazenados em divisórias do barracão de concreto e em silo. Uma carregadeira era utilizada para movimentação e abastecimento do vagão forrageiro, acoplado a um trator, utilizado para fornecer o alimento no cocho.

As dietas visavam garantir os níveis nutricionais adequados para o ganho de peso dos bovinos. Buscou-se a formulação para o menor custo de arroba produzida. Dessa forma, forneceram-se três tipos de dieta aos animais (Tabela 1). O gado recém-chegado recebia, durante 14 dias, a dieta de "Adaptação". Em seguida, foi fornecido a dieta de "Crescimento" durante cerca de 40 dias. Finalmente, até o final do ciclo de 110 dias, fornecia-se a dieta de "Terminação".

Foram registradas as benfeitorias, os maquinários, os veículos e os equipamentos de maior relevância, utilizados exclusivamente na atividade do confinamento. As depreciações dos bens foram calculadas pelo método linear (Hoffmann, 1987), em que estes se depreciam ao longo de sua vida útil de modo constante, considerandose o valor inicial, o valor final (residual) e a vida útil total dos bens. O custo variável foi obtido pelo somatório das despesas que variaram proporcionalmente de acordo com o nível de produção ou atividades, sem distinguir de custos semi-variáveis (Tabela 2) e somado aos custos fixos desembolsáveis (Tabela 3). A taxa de juros sobre o capital de giro foi nominal, no valor $8,26 \%$, correspondente a um fundo de renda fixa de 2015. 
Tabela 1. Custo e quantidade de alimento fornecido ao gado nas diferentes dietas durante o ciclo de engorda em confinamento

\begin{tabular}{|c|c|c|c|c|}
\hline \multirow{2}{*}{ Alimento } & \multirow{2}{*}{ Custo } & \multicolumn{3}{|c|}{ Dieta } \\
\hline & & Adaptação & Crescimento & Terminação \\
\hline & -- $\mathrm{R} \$ \mathrm{~kg}^{-1}--$ & ---or & Matéria Seca anim & -1 --------------------- \\
\hline Sorgo Moído & 0,38 & 2,48 & 3,34 & 3,91 \\
\hline Farelo de amendoim & 0,91 & 1,06 & 1,08 & 0,77 \\
\hline Núcleo confinamento & 2,75 & 0,36 & 0,52 & 0,62 \\
\hline Gordura Protegida & 2,64 & - & 0,07 & 0,28 \\
\hline Polpa de citrus peletizada & 0,33 & 0,97 & 2,67 & 3,49 \\
\hline Caroço de algodão & 0,73 & 0,88 & 1,03 & 1,06 \\
\hline Bagaço de cana in natura & 0,10 & 1,54 & 1,57 & 1,15 \\
\hline Total & & 7,29 & 10,28 & 11,28 \\
\hline
\end{tabular}

Tabela 2. Especificação dos itens dos componentes do custo variável

\begin{tabular}{|c|c|c|}
\hline Especificação & $\begin{array}{l}\text { Custo } \\
\text { Unitário }\end{array}$ & Custo Total \\
\hline & \multicolumn{2}{|c|}{------ R\$ animal-1 ------- } \\
\hline \multicolumn{3}{|l|}{ Aquisição de animais } \\
\hline Compra de animais & $1.742,53$ & 4.493.996,11 \\
\hline $\begin{array}{l}\text { Comissão de compra de } \\
\text { animais }\end{array}$ & 9,88 & $25.493,00$ \\
\hline Frete com os animais & 38,67 & $99.722,99$ \\
\hline \multicolumn{3}{|l|}{ Alimentação } \\
\hline Ração e afins & 576,99 & 1.488.058,59 \\
\hline Sal mineral & 4,07 & $10.495,50$ \\
\hline Silos secador & 11,28 & $29.086,45$ \\
\hline Frete dos alimentos & 6,06 & $15.621,58$ \\
\hline \multicolumn{3}{|l|}{ Higiene e Sanidade } \\
\hline Exames laboratoriais & 0,18 & 455,68 \\
\hline Vacinas e medicamentos & 2,22 & $5.717,76$ \\
\hline \multicolumn{3}{|l|}{ Máquinas } \\
\hline Horas máquinas alugadas & 33,65 & $86.791,58$ \\
\hline $\begin{array}{l}\text { Manutenção de máquinas e } \\
\text { frota }\end{array}$ & 3,29 & $8.477,32$ \\
\hline Combustíveis & 5,29 & $13.650,00$ \\
\hline Lubrificantes e filtros & 0,15 & 378,15 \\
\hline \multicolumn{3}{|l|}{ Serviços } \\
\hline Manutenção de benfeitorias & 3,34 & $8.603,03$ \\
\hline $\begin{array}{l}\text { Manutenção de } \\
\text { currais/cochos }\end{array}$ & 4,05 & $10.453,79$ \\
\hline $\begin{array}{l}\text { Manutenção de tanques e } \\
\text { bebedouros }\end{array}$ & 0,11 & 288,48 \\
\hline Mão de obra de terceiros & 6,80 & $17.537,88$ \\
\hline \multicolumn{3}{|l|}{ Demais despesas } \\
\hline Rastreabilidade & 2,81 & $7.255,12$ \\
\hline $\begin{array}{l}\text { Acordo de negociação do } \\
\text { preço do Boi Gordo }\end{array}$ & 25,18 & $64.928,80$ \\
\hline Viagem e alimentação & 7,08 & $18.262,09$ \\
\hline Curso e treinamento & 1,18 & $3.050,00$ \\
\hline Total & 95,33 & $6.408 .323,90$ \\
\hline
\end{tabular}

Tabela 3. Especificação dos itens dos componentes do custo fixo desembolsável

\begin{tabular}{lr}
\hline \multicolumn{1}{c}{ Especificação } & \multicolumn{1}{c}{$\begin{array}{c}\text { Custo Fixo } \\
\text { desembolsável }\end{array}$} \\
\hline Salários & --- R $\$$ ano $^{-1}--$ \\
Mão de obra & \\
Encargos - Folha de pagamento & $337,880,86$ \\
Despesas diversas & \\
Assessoria pecuária & $39.802,36$ \\
Telefonia Fixa & 314,70 \\
Telefonia Móvel & $2.084,83$ \\
Energia elétrica & $3.433,41$ \\
Material de escritório & 565,49 \\
Impostos & \\
Impostos $\left(\right.$ ITR $^{1}$ e IPVA ${ }^{2}$ ) & $2.868,97$ \\
\hline Total & $263.287,757$ \\
\hline
\end{tabular}

Nota: ${ }^{1}$ ITR: Imposto sobre a Propriedade Territorial Rural; ${ }^{2}$ IPVA: Imposto sobre a Propriedade de Veículos Automotores

A análise econômica foi feita com base no método de custo total de produção, que envolve os custos fixos e variáveis (Reis, 1986) e no método do custo operacional (Matsunaga et al., 1976). Utilizou-se, para os cálculos da margem bruta, margem líquida e ponto de equilíbrio, o método proposto por Reis (1986).

Os indicadores econômicos (Tabela 4) foram comparados por análises descritivas e agrupados em tabelas, objetivando melhor discussão e apresentação dos resultados.

\section{Resultados e Discussão}

A distinção entre os componentes de custo de produção associados à atividade, é de fundamental importância para assegurar que estratégias de manejo sejam aplicadas no momento ideal objetivando o melhor retorno da atividade. A Tabela 5 representa a contribuição de cada item de 
despesa no custo operacional efetivo da atividade e compara os resultados obtidos em termos percentuais com os de outros autores. De acordo com Lopes e Sampaio (1999), o monitoramento dos custos do sistema de produção de gado de corte fica mais fácil com a divisão das despesas em grupos, auxiliando o técnico e o produtor em uma análise mais criteriosa. Dessa forma, os pontos de estrangulamento podem ser detectados mais facilmente.

Tabela 4. Descrição das variáveis utilizadas e indicadores econômicos

\begin{tabular}{|c|c|c|}
\hline Variável & Unidade & Equação \\
\hline Custo Variável [CV] & $\mathrm{R} \$$ & $\begin{array}{l}=\text { gastos com aquisição de animais }+ \text { alimentação dos animais }+ \\
\text { higiene e sanidade }+ \text { máquinas }+ \text { serviços }+ \text { mão de obra de terceiros } \\
+ \text { demais despesas }^{1}\end{array}$ \\
\hline Juros sobre o Capital de Giro & $\%$ & $\begin{array}{l}=\text { percentual correspondente aos bens da empresa transformados em } \\
\text { caixa, no período de um ano, considerando o ativo circulante }\end{array}$ \\
\hline Custo de Oportunidade & $\%$ & $=$ juros sobre o Capital de Giro \\
\hline Custo fixo desembolsável & $\mathrm{R} \$$ & $\begin{array}{l}=\text { salários }+ \text { impostos }(\text { ITR e IPVA })+\text { assessoria pecuária }+ \text { telefonia } \\
+ \text { energia elétrica }+ \text { material de escritório }\end{array}$ \\
\hline Custo Operacional Efetivo & $\mathrm{R} \$$ & $=$ custo variável + custo fixo desembolsável \\
\hline Depreciação & $\mathrm{R} \$$ & $\begin{array}{l}\text { = desvalorização das máquinas e equipamentos, calculado pelo método } \\
\text { linear, considerando valor de compra e de venda descontando o tempo } \\
\text { de utilização de acordo com a vida útil }\end{array}$ \\
\hline Custo Operacional Total & $\mathrm{R} \$$ & $=$ custo operacional efetivo + depreciação \\
\hline Juros sobre o Capital não circulante & $\mathrm{R} \$$ & $=$ valor de máquinas e implementos descontados a depreciação \\
\hline Custo Total & $\mathrm{R} \$$ & $=$ custo operacional total + juros sobre o capital não circulante \\
\hline Receita & $\mathrm{R} \$$ & $\begin{array}{l}=\text { número de animais } * \text { peso médio final } * \text { preço de venda }\left(\mathrm{R} \$ \mathrm{~kg}^{-1}\right. \\
\left.\text { animal }^{-1}\right)\end{array}$ \\
\hline Margem de Contribuição & $\mathrm{R} \$$ & $=$ custo variável - receita \\
\hline Índice de Margem de Contribuição & $\%$ & $=$ margem de contribuição $/$ receita \\
\hline Margem de Contribuição Operacional Efetiva & $\mathrm{R} \$$ & $=$ receita menos - operacional efetivo \\
\hline Lucro Operacional & $\mathrm{R} \$$ & $=$ Receita - custo operacional total \\
\hline Lucro Líquido na Atividade & $\mathrm{R} \$$ & $=$ Receita - custo total \\
\hline Margem de Lucro & $\%$ & = Lucro líquido na atividade dividido / receita \\
\hline Ponto de Equilíbrio & $\mathrm{kg}$ & $\begin{array}{l}\text { = Quantidade de quilogramas de carne produzida em que a receita se } \\
\text { iguala ao custo fixo mais variável }\end{array}$ \\
\hline
\end{tabular}

Nota: ${ }^{1}$ Sorgo Moído, Farelo de amendoim, Núcleo confinamento, Gordura Protegida, Polpa de citrus peletizada, Caroço de algodão e Bagaço de cana in natura

O custo operacional efetivo representa o desembolso médio feito pelo produtor para custear a atividade. Os itens que mais contribuíram para o custo operacional efetivo (considerando custo fixo desembolsável e custo variável, exceto juros sobre capital de giro) foram a aquisição de animais, com cerca de $\mathrm{R} \$$ 4,6 milhões (72,08\%), e a alimentação, com aproximadamente $\mathrm{R} \$$ 1,5 milhão (24,08\%). Estes corresponderam a 96,16\% do custo operacional efetivo. Dentre os alimentos que compunham a dieta, os gastos com volumoso e concentrado representaram, respectivamente, 15\% e 85\% (Tabela 1). Esses resultados demonstram a importância de conhecer a variação dos preços de compra de animais e de alimentação, sendo de fundamental importância para gestão e maior lucro na atividade (Barbieri et al., 2016). As variações impostas pelo mercado sobre o valor do animal para abate e preço dos insumos, principalmente em sistemas de terminação de bovinos em confinamento, determinam a rentabilidade e a viabilidade da atividade (Santos et al., 2017).

Os resultados demonstrados na Tabela 5 foram corroborados por diferentes autores. Nogueira (2007) relatou que a aquisição de animais representa participação de $64,56 \%$ e que a alimentação contribui com $28,67 \%$. Coan (2015) reporta valores próximos a $70 \%$ para a aquisição de animais e de cerca de $20 \%$ para a alimentação. Lopes e Sampaio (1999) e Lopes e Magalhães (2005) obtiveram valores de $67,87 \%$ e $68,41 \%$, respectivamente, para a aquisição de animais, e 29,84\% e 22,32\% para alimentação. Neste sistema de terminação em confinamento, o boi magro foi adquirido pelo preço médio de $\mathrm{R} \$ 1.742,53$, média abaixo da observada no período de janeiro a setembro de 2015, para as regiões de Minas Gerais (R \$ 1.751,11), Goiás $(\mathrm{R} \$ 1.867,22)$ e São Paulo ( $\$$ 1.937,22) (BeefPoint, 2015). 
Tabela 5. Contribuição de cada item no custo operacional efetivo em sistema de engorda de bovinos em confinamento comparado com outros estudos

\begin{tabular}{|c|c|c|c|c|c|c|}
\hline Itens & Despesas & Estudo & $\begin{array}{c}\text { Lopes e Sampaio } \\
\text { (1999) }\end{array}$ & $\begin{array}{l}\text { Lopes e Magalhães } \\
\qquad(2005)\end{array}$ & $\begin{array}{l}\text { Coan } \\
(2015)\end{array}$ & $\begin{array}{l}\text { Nogueira } \\
(2007)\end{array}$ \\
\hline & ------ R\$ mil ------- & 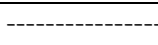 & --------------------- & - $\%$ - & - & ------------- \\
\hline Animais & $4.619,21$ & 72,08 & 67,87 & 68,41 & 72,61 & 64,56 \\
\hline Alimentação & $1.543,26$ & 24,08 & 29,84 & 22,32 & 20,79 & 28,67 \\
\hline Outras Despesas ${ }^{1}$ & 93,46 & 1,45 & 1,04 & 4,32 & - & - \\
\hline Mão-de-obra ${ }^{2}$ & 36,88 & 0,57 & 0,51 & 3,90 & 4,99 & 6,55 \\
\hline Sanidade & 6,17 & 0,10 & 0,74 & 0,93 & 0,12 & 0,21 \\
\hline Impostos & 2,87 & 0,04 & - & 0,12 & - & - \\
\hline Frete & 99,72 & 1,80 & - & - & 1,49 & - \\
\hline Total & $6.671,61$ & 100,00 & 100,00 & 100,00 & 100,00 & 100,00 \\
\hline
\end{tabular}

Nota: ${ }^{1}$ Rastreabilidade; Acordo de negociação de preço do Boi Gordo; Viagem e alimentação; Curso e treinamento. ${ }^{2}$ Incluindo o somatório de serviços e mão-de-obra de terceiro

É interessante enfatizar que os gastos com higiene e sanidade, $R \$ 6.173,44$, foram baixos, representando $0,09 \%$ dos custos. Dessa forma, não se justificaria deixar de vacinar ou realizar o controle de parasitas, pois os prejuízos decorrentes dessas doenças poderiam influenciar significativamente o resultado da atividade. Em gados vacinados, a conversão alimentar aumentou em 2,1\% comparativamente a gados não vacinados (Cull et al., 2012), com impacto significativo sobre os resultados econômicos (Tonson e Schroeder, 2015).

A receita bruta representa o total obtido pelas vendas, sendo considerado nesse estudo apenas a venda dos animais para o frigorífico ao final do período de terminação. Dessa forma, a receita bruta foi obtida considerando a média do preço da arroba negociada com um frigorífico da região e a quantidade de peso final obtida por animal (Tabela 6). O peso médio de saída dos animais ao final do ciclo (110 dias) foi de $530,92 \mathrm{~kg}$. A média do ganho de peso diário foi de $1,35 \mathrm{~kg} \mathrm{dia}{ }^{-1}$. Obteve-se um rendimento de carcaça de $56,45 \%$, valor considerado alto quanto se compara à média dos frigoríficos no Brasil, que é de $52 \%$ para bois engordados no pasto e $54 \%$ para animais terminados em confinamento (Jurca, 2014).

Tabela 6. Receita bruta obtida de acordo com o peso final, rendimento de carcaça e preço da arroba de animais engordados em regime de confinamento por um período de 110 dias

\begin{tabular}{|c|c|c|c|c|c|c|}
\hline Número de animais & $\begin{array}{c}\text { Peso } \\
\text { médio inicial }\end{array}$ & $\begin{array}{c}\text { Peso } \\
\text { médio final }\end{array}$ & $\begin{array}{l}\text { Rendimento } \\
\text { médio de carcaça }\end{array}$ & $\begin{array}{l}\text { Quantidade } \\
\text { animal }\end{array}$ & Preço & Receita Bruta \\
\hline & \multicolumn{2}{|c|}{-------------- kg --------------- } & --------- \% --------- & ----- kg ------ & $--\mathrm{R} \$ \mathrm{~kg}^{-1}---$ & ---- R\$ --- \\
\hline 2.579 & 382,00 & 530,92 & 56,45 & 299,70 & 10,23 & 7.910.191,69 \\
\hline
\end{tabular}

O preço da arroba negociada no período foi de $\mathrm{R} \$ 153,52$. Em consulta à série histórica do Centro de Estudos Avançados em Economia Aplicada [CEPEA] (2018), constatou-se que a média para a região onde os bois foram vendidos foi de $\mathrm{R} \$ 148,48$ no segundo semestre de 2015 . O pecuarista travou antecipadamente o preço de venda com o frigorífico no mercado a termo, e dessa forma conseguiu valores acima da média local. Assim, com a venda de 2.579 animais, obteve-se uma receita bruta de $\mathrm{R} \$$ 7.910.191,69.

O esterco produzido pelo sistema de terminação de bovinos em confinamento não era vendido ou aproveitado em outra atividade da fazenda, como adubação orgânica ou produção de energia. Lopes e Magalhães (2005) relataram que a venda de esterco representou $0,56 \%$ do faturamento da atividade e citam que Lopes e Sampaio (1999) encontraram valores de participação na receita de 2,6\%, podendo, portanto, incrementar de $\mathrm{R} \$ 44.546,53$ a $\mathrm{R} \$ 206.823,175$ na receita, caso o esterco fosse vendido.

A depreciação refere-se a inclusão dos abatimentos de valores advindos do desgaste efetivo dos bens pelo uso ou perda da utilidade, mesmo que seja por ação do tempo ou por simples obsolescência. A Tabela 7 apresenta as benfeitorias, as máquinas e os equipamentos utilizados na atividade do confinamento em estudo. Com o valor inicial total ( $\mathrm{R} \$ 2.298 .000,00)$ e o valor residual calculou-se a depreciação $(\mathrm{R} \$ 98.246,67)$ pelo método linear. 
Tabela 7. Valores iniciais, vida útil, valores residuais e valores de depreciação de instalações, máquinas, equipamentos e benfeitorias utilizados exclusivamente na atividade de confinamento de 2.579 bovinos

\begin{tabular}{|c|c|c|c|c|c|}
\hline Especificação & Quantidade & Valor inicial & Vida útil & Valor residual & Valor depreciação \\
\hline & & ------ R\$ ------- & --- Anos --- & ------- \% ------- & ---------- R\$ --------- \\
\hline Pá carregadeira & 1 & $67.000,00$ & 10 & 10 & $6.030,00$ \\
\hline Vagão Forrageiro & 1 & $100.000,00$ & 10 & 10 & $9.000,00$ \\
\hline Trator & 1 & $70.000,00$ & 10 & 10 & $6.300,00$ \\
\hline Veículo & 1 & $31.000,00$ & 10 & 10 & $2.790,00$ \\
\hline Barracão & 1 & $400.000,00$ & 30 & 20 & $10.666,67$ \\
\hline Curral de Manejo & 1 & $80.000,00$ & 25 & 20 & $2.560,00$ \\
\hline Silo secador de grãos & 1 & $300.000,00$ & 15 & 10 & $18.000,00$ \\
\hline Reservatório de água & 1 & $50.000,00$ & 10 & 10 & $4.500,00$ \\
\hline Instalação do confinamento & 1 & $1.200 .000,00$ & 25 & 20 & $38.400,00$ \\
\hline Total & & $2.298 .000,00$ & & & $98.246,67$ \\
\hline
\end{tabular}

O valor referente à depreciação ( $\mathrm{R}$ 98.246,67), embora não seja um desembolso, representa uma reserva de caixa que deve estar disponível quando houver necessidade de se repor os bens patrimoniais (benfeitorias, máquinas, equipamentos etc.) ao final de sua vida útil. Assim, o pecuarista teria recursos monetários para efetuar a reposição sem se descapitalizar. A depreciação correspondeu a $1,34 \%$ do custo operacional total, acima do relatado por Moreira et al. (2009), que obteve 1,05\%, e abaixo o relatado por Lopes e Sampaio (1999) e Lopes e Magalhães (2005), cujos valores foram 1,6\% e 1,7\%, respectivamente (Tabela 7).

A Tabela 8 apresenta a discriminação dos custos de produção do sistema de terminação de bovinos em

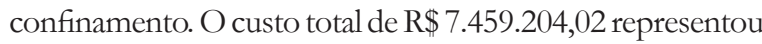
a soma dos custos fixos e dos custos variáveis. O custo variável representa os custos que mudam de acordo com o nível de produção ou quantidade de trabalho, e representou nesse estudo 93,3 \% do custo total. A elevada influência na participação dos custos dos componentes aquisição de animais e alimentação reforça a importância no controle desses fatores por meio de contratos de fixação de preços e manejo adequado do sistema para otimizar o uso desses insumos e nível de produtividade (Asimwe et al., 2016). Adicionalmente, Lopes et al. (2018) reportam que a gestão nutricional é uma importante ferramenta a ser utilizada para otimizar os índices econômicos em períodos de restrição alimentar, particularmente durante os períodos de aumento dos preços dos insumos.

O custo operacional total, $\mathrm{R} \$ 7.320 .933,44$, foi obtido pela soma do custo operacional efetivo, representado pelo desembolso de dinheiro para custear a atividade, e do custo de depreciação dos bens patrimoniais. Considerou-se uma taxa de rentabilidade anual de $8,26 \%$ (correspondente a um fundo de renda fixa de 2015) para se obter o custo fixo do capital e juros sobre o capital não circulante (horizonte de tempo de 20 anos).

$\mathrm{Na}$ Tabela 9 observa-se os índices econômicos do sistema de terminação de bovinos em confinamento. $\mathrm{O}$ custo de produção foi de $\mathrm{R} \$ 9,65 \mathrm{~kg}^{-1}$, valores superiores aos maiores custos totais para os confinamentos do Estado de São Paulo, que foram de $R$ \$ 9,17 $\mathrm{kg}^{-1}$ e $\mathrm{R} \$ 9,08 \mathrm{~kg}^{-1}$ para fazendas de médio e grande porte, respectivamente (Sartorello et al., 2018). As margens brutas e líquidas refletem a eficiência econômica da atividade, e determinam a rentabilidade. Os valores positivos de margem bruta (ou margem de contribuição operacional efetiva), de R\$ 687.504,91, e margem líquida (lucro operacional), de $\mathrm{R} \$$ $589.258,25$, indicam que a atividade de confinamento teve condições de se manter no médio e longo prazo, respectivamente. Ferraza et al. (2015) reportaram valores inferiores, de $\mathrm{R} \$ 12.287,51$ para margem bruta e negativo de $\mathrm{R} \$$ - 50.822,03 para margem líquida, mostrando-se insatisfatório no médio e longo prazo para a atividade de terminação de bovinos de corte em Minas Gerais, Brasil. $\mathrm{O}$ valor obtido para margem líquida pode ser comparado, por exemplo, com a caderneta de poupança ou qualquer outra opção de investimento, o que confere maior poder de comparação (Lopes et al., 2011).

O lucro líquido da atividade (resultado), que corresponde à receita menos o custo total, foi de $\mathrm{R} \$$ $450.987,67$, correspondente a uma margem de lucro de $5,7 \%$. A margem de lucro indica o ganho obtido sobre as vendas realizadas, de forma que quanto maior, mais o negócio está justificando a operação, ou seja, as vendas são suficientes para pagar os custos e despesas e ainda gerar lucro. $\mathrm{O}$ índice de margem de contribuição, que significa, em termos porcentuais, quanto do total das vendas contribui para cobrir o total do custo fixo e proporcionar lucro, foi $12,02 \%$ (Tabela 9). 
Tabela 8. Discriminação dos custos de produção da atividade de engorda de 2.579 bovinos de corte terminados em confinamento

\begin{tabular}{|c|c|c|c|c|c|}
\hline \multirow{3}{*}{ Componentes do custo } & \multirow{2}{*}{\multicolumn{2}{|c|}{ Despesas }} & \multicolumn{3}{|c|}{ Participação } \\
\hline & & & \multirow{2}{*}{$\mathrm{COE}$} & \multirow{2}{*}{ COT } & \multirow{2}{*}{ CT } \\
\hline & ------ R\$ mil ------ & ---- $\mathrm{R} \$ \mathrm{~kg}^{-1}$---- & & & \\
\hline Custo variável & $6.959,40$ & 9,06 & 96,35 & 95,06 & 93,30 \\
\hline Mão de Obra & 17,54 & 0,02 & 0,24 & 0,24 & 0,24 \\
\hline Aquisição de animais & $4.494,00$ & 5,81 & 62,22 & 61,39 & 60,25 \\
\hline Alimentação & $1.498,55$ & 1,94 & 20,75 & 20,47 & 20,09 \\
\hline Higiene e sanidade & 6,17 & 0,08 & 0,09 & 0,08 & 0,08 \\
\hline Frete & 99,72 & 0,13 & 1,38 & 1,36 & 1,34 \\
\hline Despesas diversas ${ }^{1}$ & 292,34 & 0,40 & 4,05 & 3,99 & 3,92 \\
\hline Juros sobre capital de Giro & 551,08 & 0,71 & 7,63 & 7,53 & 7,39 \\
\hline Custo Fixo Desembolsável Total & 263,29 & 0,34 & 3,65 & 3,60 & 3,53 \\
\hline Salários & 214,22 & 0,28 & 2,97 & 2,93 & 2,87 \\
\hline Despesas diversas & 46,20 & 0,06 & 0,64 & 0,63 & 0,62 \\
\hline Impostos (ITR e IPVA)* & 2,87 & 0,004 & 0,04 & 0,04 & 0,04 \\
\hline Custo Operacional Efetivo [COE] & $7.222,69$ & 9,34 & 100,00 & 98,66 & 96,83 \\
\hline Depreciação & 98,25 & 0,13 & - & 1,34 & 1,32 \\
\hline Custo Operacional Total [COT] & $7.320,93$ & 9,47 & - & 100,00 & 98,15 \\
\hline Juros sobre capital não circulante & 138,27 & 0,18 & - & 1,89 & 1,85 \\
\hline Custo Total [CT] & $7.459,20$ & 9,65 & - & - & 100,00 \\
\hline
\end{tabular}

Nota: ${ }^{*}$ ITR: Imposto sobre a Propriedade Territorial Rural; IPVA: Imposto sobre propriedade de veículo automotor; ${ }^{1}$ Detalhados na Tabela 2.

Tabela 9. Estimativa da receita e indicadores de rentabilidade da atividade de terminação de 2.579 bovinos de corte em confinamento

\begin{tabular}{|c|c|c|c|}
\hline \multirow{2}{*}{ Especificação } & \multirow{2}{*}{ Unidade } & \multicolumn{2}{|c|}{ Valores } \\
\hline & & Total & Unitário \\
\hline & & & ------ R\$ kg-1 ------ \\
\hline Receita & $\mathrm{R} \$$ mil & $7.910,19$ & 10,23 \\
\hline Custo Total & $\mathrm{R} \$$ mil & $7.459,20$ & 9,65 \\
\hline Custo Variável & $\mathrm{R} \$$ mil & $6.959,40$ & 9,00 \\
\hline Margem de Contribuição & $\mathrm{R} \$$ mil & 950,79 & 1,23 \\
\hline Índice de Margem de Contribuição & $\%$ & 12,02 & - \\
\hline Margem de Contribuição Operacional efetiva & $\mathrm{R} \$$ mil & 687,50 & 0,89 \\
\hline Margem de Contribuição Operacional efetiva & $\%$ & 8,69 & - \\
\hline Ponto de Equilíbrio - peso animal & $\mathrm{kg}$ mil & 729,15 & - \\
\hline Lucro Operacional & $\mathrm{R} \$$ mil & 589,26 & 0,76 \\
\hline Lucro líquido da atividade & $\mathrm{R} \$$ mil & 450,99 & 0,58 \\
\hline Margem de Lucro & $\%$ & 5,70 & - \\
\hline
\end{tabular}

Esses índices demonstram o potencial da atividade de confinamento para a realização de investimentos e geração de fluxo de caixa. Ademais, a pecuária de corte é uma atividade ampla, sendo possível agregar valor por meio de fatores como produção de carne de qualidade (Venkata Reddy et al., 2015), otimizar o retorno financeiro em função de estratégias alimentares adaptadas a fase do animal (Lopes et al., 2018), adaptar o nível de intensificação de produção de acordo com a categoria animal (Asizua et al., 2017), entre outros.

\section{Conclusão}

A análise dos índices econômicos mostra que o resultado final do sistema de terminação de bovinos em confinamento é positivo. Os componentes do custo operacional efetivo que exercem maior influência sobre os custos da atividade de confinamento é a aquisição dos animais e a alimentação. Os índices econômicos apontam que a atividade é lucrativa e está se remunerando em curto prazo. 


\section{Referências}

Associação Nacional dos Confinadores [ASSOCON]. 2012. Levantamento da Assocon sobre o sistema de produção em confinamento no Brasil. Disponível em: <http://www. beefpoint.com.br/cadeia-produtiva/giro-do-boi/assocondivulga-i-censo-nacional-de-confinadores-de-2010-2011-eestimativas-para-2012/>. Acesso em: 10 set. 2016.

Albright, M.L.; Schroeder, T.C.; Langemeier, M.R.; Mintert, J.R.; Brazle, F.; Luther, L.W.; Johnson, Z.B. 1993. Cattle feeding profit and cost of gain variability determinants. The Professional Animal Scientist 9: 138-145.

Asimwe, L.; Kimambo, A.E.; Laswai, G.H.; Mtenga, L.A.; Weisbjerg, M.R.; Madsen, J. 2016. Economics of finishing Tanzania shorthorn zebu cattle in feedlot and optimum finishing period. Livestock Research for Rural Development 28: 1-11.

Asizua, D.; Mpairwe, D.; Kabi, F.; Mutetikka, D.; Hvelplund, T.; Weisbjerg, M.R.; Madsen, J. 2017. Effects of grazing and feedlot finishing duration on the performance of three beef cattle genotypes in Uganda. Livestock Science 199: 25-30. doi:10.1016/j.livsci.2017.03.006.

Barbieri, R.S.; Carvalho, J.B.; Sabbag, O.J. 2016. Análise de viabilidade econômica de um confinamento de bovinos de corte. Interações 17(3): 357-369.

Beefpoint. 2015. Arquivo da Tag: Boi magro. <Disponível em: http://www.beefpoint.com.br/tag/boi-magro/>. Acesso em: 15 out. 2016

Centro de Estudos Avançados em Economia Aplicada [CEPEA]. 2018. Preço do boi gordo. Disponível em: <http://www.cepea.esalq.usp.br/br/indicador/boi-gordo. aspx>. Acesso em: 16 jun. 2018

Coan, R. 2015. Viabilidade do confinamento em 2015. Disponível em: <http://www.beefpoint.com.br/cadeiaprodutiva/viabilidade-confinamento-em-2015-avaliacaoparcial-1o-semestre/>. Acesso em: 13 out. 2016.

Cull, C.A.; Paddock, Z.D.; Nagaraja, T.G.; Bello, N.M.; Babcock, A.H.; Renter, D.G. 2012. Efficacy of a vaccine and directfed microbial against fecal shedding of Escherichia coli O157:H7 in a randomized pen-level field trial of commercial feedlot cattle. Vacccine 30: 6210-6215.

Ferrazza, R.A.; Lopes, M.A.; Santos, T.K. 2015. Profitability analysis of the finishing of beef cattle in feedlot: a case study in 2009, in the western region of Minas Gerais, Brazil. Archivos Latinoamericanos de Producción Animal 23: $37-$ 47.

Hoffmann, R. 1987. Administração da empresa agrícola. Livraria Pioneira, São Paulo, SP, Brasil.

Jurca, P. 2014. Rendimento de carcaça em frigoríficos do Brasil. Disponível em: <https://www.scotconsultoria. com.br/noticias/artigos/37616/rendimento-de-carcacaem-frigorificos-do-brasil-.htm>. Acesso em: 13 out. 2016.

Kamali, F.P.; Linden, A.; Meuwissen, M.P.M.; Malafaia, G.C.; Lansink, A.G.J.M.O.; Boer, I.J.M. 2016. Environmental and economic performance of beef farming systems with different feeding strategies in southern Brazil. Agricultural Systems 146:70-79. https://doi.org/10.1016/j. agsy.2016.04.003

Koknaroglu, H.; Loy, D.D.; Wilson, D.E.; Hoffman, M.P.; Lawrence, J.D. 2005. Factors affecting beef cattle performance and profitability. The Professional Animal Scientist 21: 286-296.

Lobato, J.F.P.; Freitas, A.K.; Devincenzi, T.; Cardoso, L.L.; Tarouco, J.U.; Vieira, R.M.; Dillenburg, D.R.; Castro, I. 2014. Brazilian beef produced on pastures: sustainable and healthy. Meat Science 98:336-345. https://doi.org/10.1016/j. meatsci.2014.06.022

Lopes, R.B.; Canozzi, M.E.A.; Canellas, L.C.; Gonzalez, F.A.L.; Corrêa, R.F.; Pereira, P.R.R.X.; Barcellos, J.O.J. 2018. Bioeconomic simulation of compensatory growth in beef cattle production systems. Livestock Science 216: 165-173. doi:10.1016/j.livsci.2018.08.011.

Lopes, L.S.; Ladeira, M.M.; Machado Neto, O.R.; Silveira, A.R.M.C.; Reis, R. R.; Campos, F.R. 2011. Viabilidade econômica da terminação de novilhos Nelore e Red Norte em confinamento na região de Lavras-MG. Ciência e Agrotecnologia 35:774-780. https://doi.org/10.1590/ S1413-70542011000400017

Lopes, M.A.; Sampaio, A.A.M. 1999. Manual do confinador de bovinos de corte. FUNEP. Jaboticabal, SP, Brasil.

Lopes, M.A.; Magalhães, G.P. 2005. Rentabilidade na terminação de bovinos de corte em condições de confinamento: um estudo de caso em 2003, na região oeste de Minas Gerais. Ciência e Agrotecnologia 29(5): 1039-1044.

Matsunaga, M.; Bemelmans, P.F.; Toledo, P.E.N. 1976. Metodologia de custo de produção utilizado pelo IEA. Agricultura em São Paulo 23(1) 123-139.

Moreira, S.A.; Thomé, K.M.; Ferreira, P.S.; Botelho Filho, F.B. 2009. Análise econômica da terminação de gado de corte em confinamento dentro da dinâmica de uma propriedade agrícola. Custose@gronegócio online 5 (3) 132-152.

Nogueira, M.P. 2007. Custos e Viabilidade do Confinamento Frente aos Preços Baixos. In: Encontro de Confinamento: Gestão técnica e Econômica. Jaboticabal, SP, Brasil. Disponível em: <http://www.coanconsultoria.com.br/ images/palestras/Custos \%20e $\% 20$ viabilidade $\% 20 \mathrm{do} \% 20$ confinamento.pdf $>$. Acesso em: 8 set. 2016.

Peixoto, A.M.; Haddad, C.M.; Boin, C.; Bose, M.L.V. 1989. O confinamento de bois. Globo. São Paulo, SP, Brasil.

Reis, D.L. 1986. Estudo técnico e econômico da propriedade rural. Informe Agropecuário 12 (143): 23-38.

Santos, P.B.; Santana Junior, H.A.; Araújo, M.J.; Oliveira, A.P.; Freitas, T.B.; Viana, P.T. 2017. Production and economic viability of feedlot beef cattle categories. Acta Scientiarum. Animal Sciences 39: 195-199. doi:10.4025/actascianimsci. v39i2.33452.

Sartorello, G.L.; Bastos, J.P.S.T.; Stivari, S.; Gameiro, A.H. 2018. Development of a calculation model and production cost index for feedlot beef cattle. Revista Brasileira Zootecnia 47: 1-11. doi:https://doi.org/10.1590/rbz4720170215.

Tang, M.; Lewis, K.E.; Lambert, D.M.; Griffith, A.P.; Boyer, C.N. 2017. Beef cattle retained ownership and profitability in Tennessee. Journal of Agricultural and Applied Economics 49: 571-591. doi:10.1017/aae.2017.12.

Tonsor, G.T.; Schroeder, T.C. 2015. Market impacts of E. Coli vaccination in U.S. Feedlot cattle. Agricultural and Food Economics 3(7): 1-15. doi:10.1186/s40100-014-00212.

Venkata Reddy, B.; Sivakumar, A.S.; Jeong, D.W.; Woo, Y.B.; Park, S.J.; Lee, S.Y.; Byun, J.; Kim, C.; Cho, S.; Hwang, I. 2015. 
Beef quality traits of heifer in comparison with steer, bull and cow at various feeding environments. Animal Science Journal 86: 1-16. doi:10.1111/asj.12266.

Wedekin, V.S.P.; Bueno, C.R.F.; Amaral, A.M.P. 1994. Análise econômica do confinamento de bovinos. Informações Econômicas 24(9): 123-31. 